\title{
Mechanism of thiol oxidation by the superoxide radical
}

\author{
Bruno CARDEY, Sarah FOLEY and Mironel ENESCU*
}

\section{Supporting information}

1. Gaussian 03 input file for the geometry optimization of the transition state TS1 at the IMOMO (QCISD+UMP2) level of theory. Includes the optimized Cartesian coordinates.

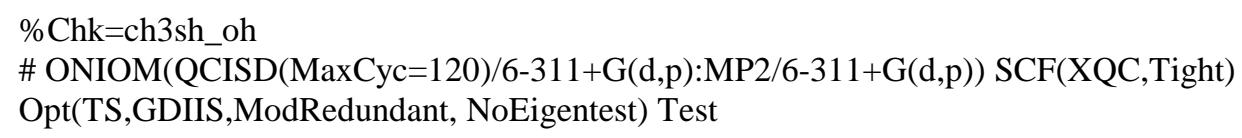

2. Gaussian 03 input file for the geometry optimization of the $\mathrm{AD}$ three electron bond adduct at the QCISD/6-311+G(d,p) level of theory. Includes the optimized Cartesian coordinates.

$\%$ Chk=AD1_qcisd

\# QCISD(MaxCyc=120)/6-311+G(d,p) SCF(XQC,Tight) Opt(GDIIS,ModRedundant,MaxStep=4) Test

AD QCISD

$\begin{array}{crrr}-12 & & & \\ \mathrm{~S} & -1.041802 & -0.668274 & -0.155466 \\ \mathrm{O} & 1.192564 & -0.427508 & 0.639062 \\ \mathrm{O} & 1.901503 & 0.388055 & -0.344592 \\ \mathrm{H} & 1.956629 & -0.231733 & -1.078419 \\ \mathrm{C} & -1.112266 & 1.123842 & 0.117055 \\ \mathrm{H} & -1.223076 & 1.361305 & 1.181251\end{array}$


$\begin{array}{llll}\mathrm{H} & -0.183427 & 1.576285 & -0.242352 \\ \mathrm{H} & -1.960238 & 1.559094 & -0.431111\end{array}$

3. Gaussian 03 input file for the geometry optimization of the transition state TS2 at the IMOMO (QCISD+UMP2) level of theory. Includes the optimized Cartesian coordinates.

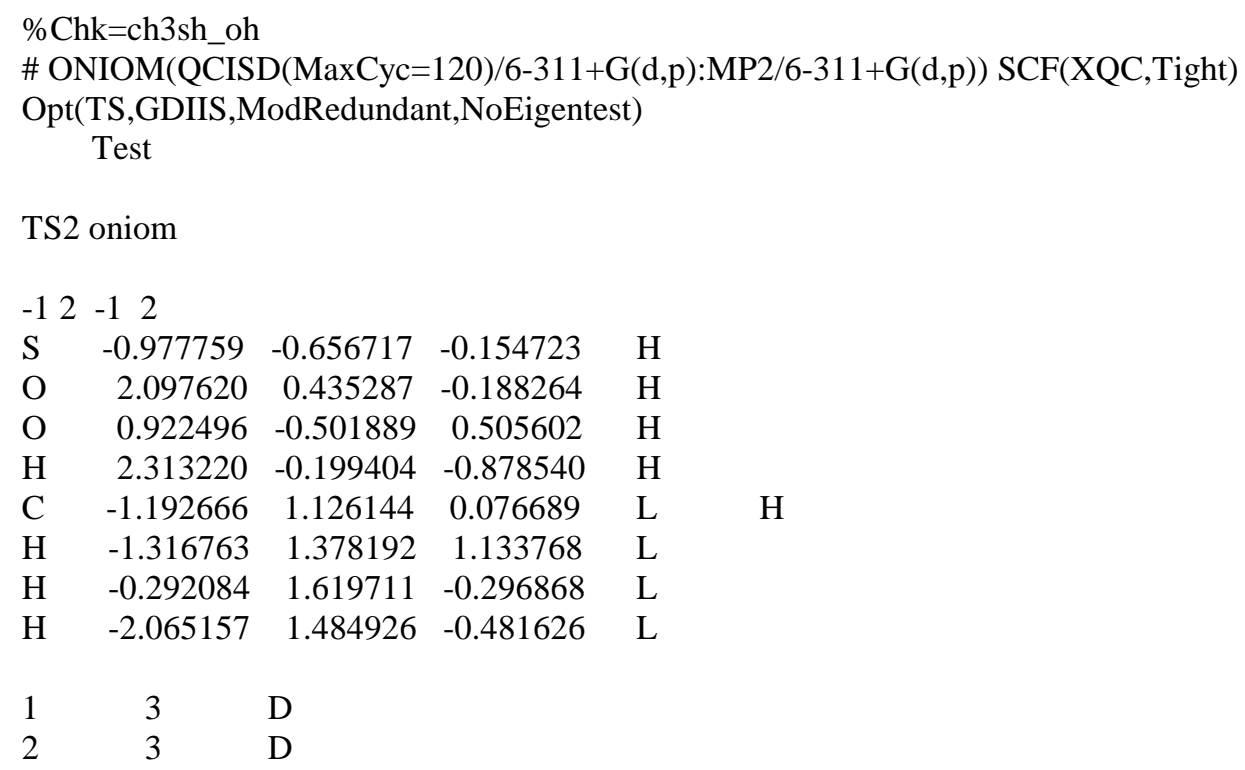

4. Gaussian 03 input file for the geometry optimization of the transition state TS2 at the QCISD/6-311+G(d,p) level of theory. Includes the optimized Cartesian coordinates.

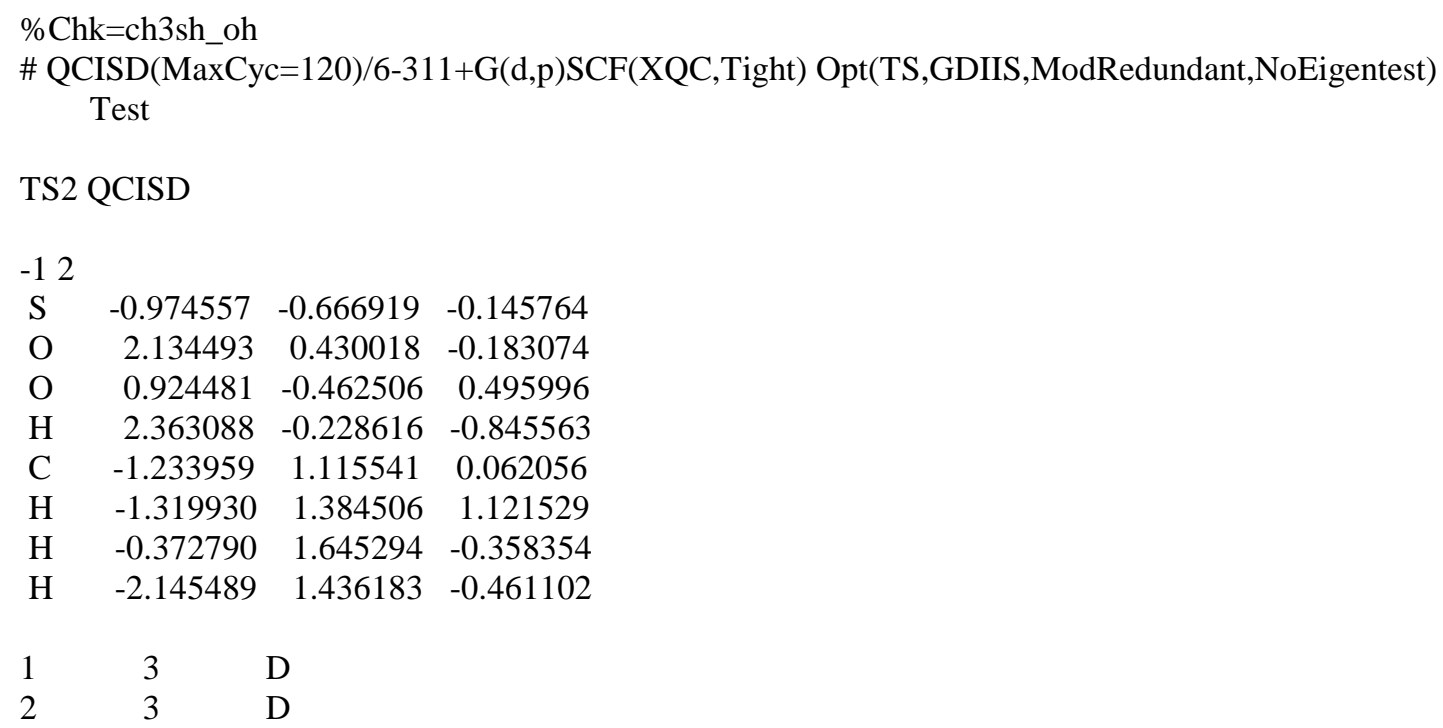

5. Gaussian 03 input file for the geometry optimization of the transition state TS1' at the IMOMO (QCISD+UMP2) level of theory. Includes the optimized Cartesian coordinates. 


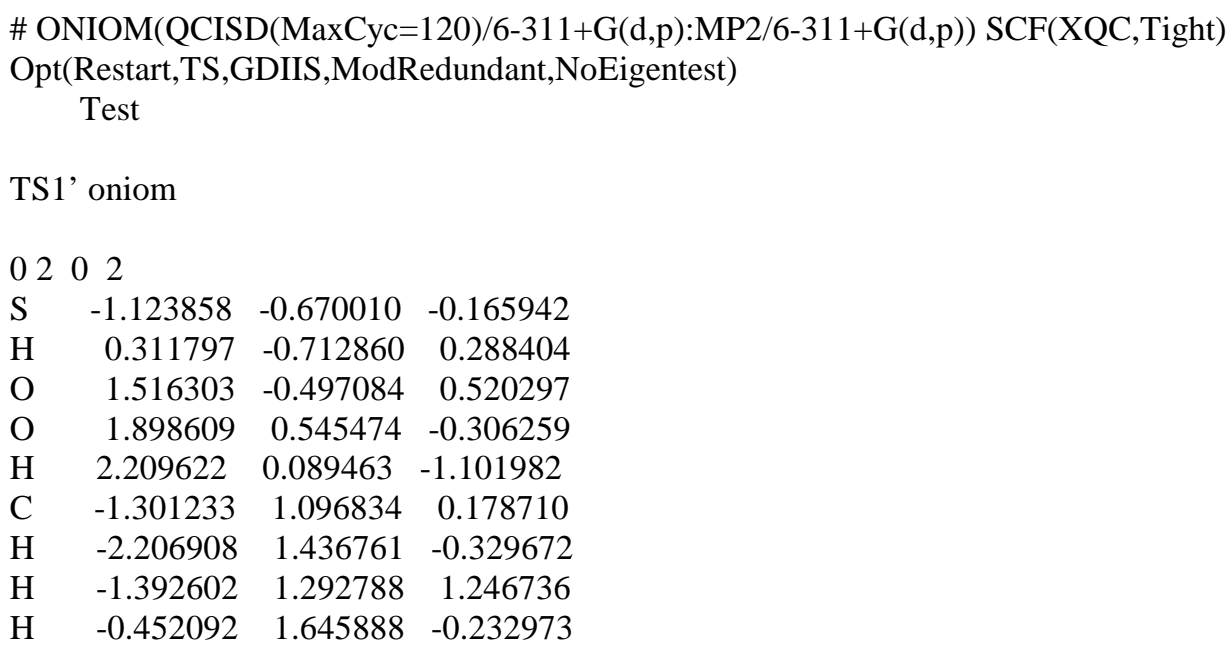

6. Gaussian 03 input file for the geometry optimization of the transition state TS2' at the

IMOMO (QCISD+UMP2) level of theory. Includes the optimized Cartesian coordinates.

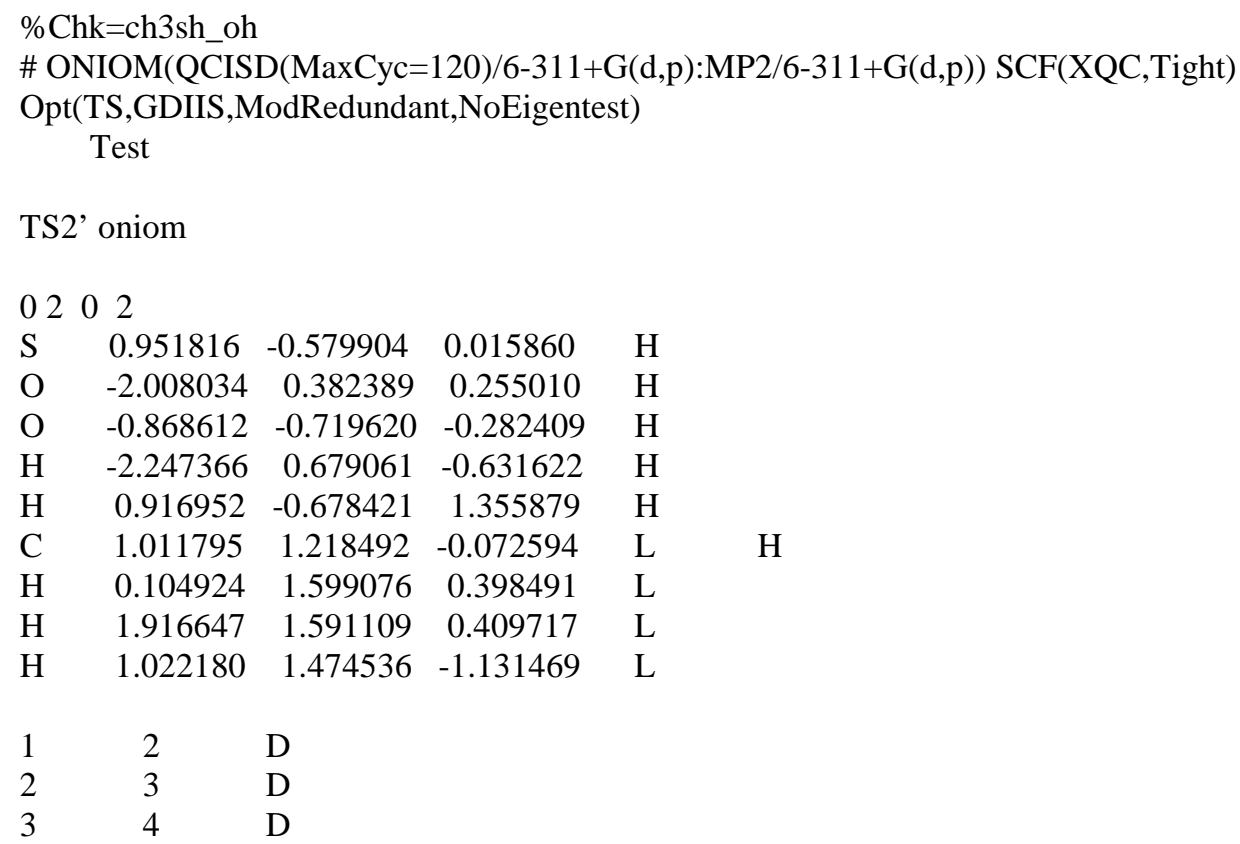

7. Gaussian 03 input file for single point energy calculation of the transition state TS 1 at the QCISD(T)/6-311+G(2df,2pd) level of theory.

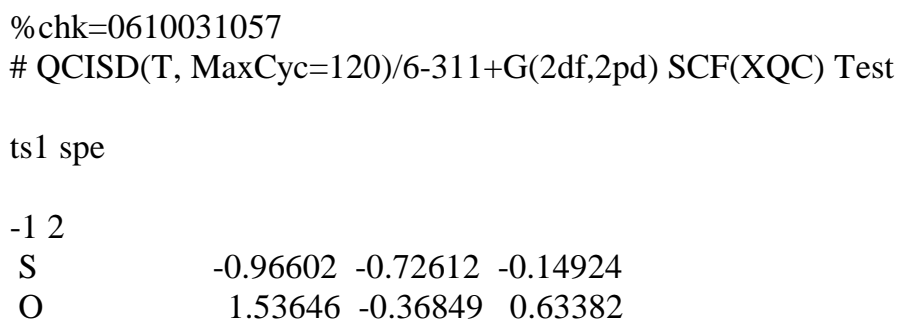




\begin{tabular}{|c|c|c|c|}
\hline $\mathrm{O}$ & 1.7792 & 0.51349 & -0.4287 \\
\hline $\mathrm{H}$ & 1.2951 & 0.04785 & -1.12981 \\
\hline C & -1.34709 & 1.02808 & 0.18386 \\
\hline $\mathrm{H}$ & -1.72728 & 1.17074 & + 1.20284 \\
\hline $\mathrm{H}$ & -0.44957 & 1.64958 & 0.07714 \\
\hline 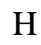 & -2.10466 & 1.42129 & -0.50651 \\
\hline
\end{tabular}

8. Gaussian 03 input file for the IPCM solvation energy calculation of the transition state TS1 at the MP2/6-311+G(d,p) level of theory.

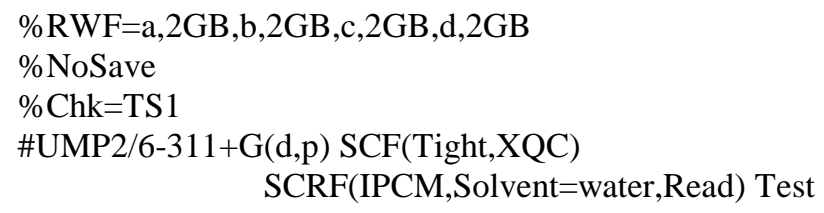

Table 1. Gas phase potential energy $\left(E_{\mathrm{p}, \mathrm{g}}\right)$ and Gibbs free energy in gas phase $\left(\mathrm{G}_{\mathrm{g}}\right)$ and aqueous solution $\left(\mathrm{G}_{\mathrm{aq}}\right)$ of free reactants and some possible products of the methanethiol oxidation by the superoxide radical anion. All energies are given in Hartree.

\begin{tabular}{|c|c|c|c|}
\hline & $\mathrm{E}_{\mathrm{p}, \mathrm{g}}$ & $\mathrm{G}_{\mathrm{g}}$ & $\mathrm{G}_{\mathrm{aq}}$ \\
\hline $\mathrm{CH}_{3} \mathrm{SH}+\mathrm{O}_{2}^{\bullet-}$ & -588.28801 & -588.28247 & -588.39067 \\
\hline $\mathrm{CH}_{3} \mathrm{SO}^{\bullet}+\mathrm{HO}^{-}$ & -588.33688 & -588.32971 & -588.45566 \\
\hline $\mathrm{CH}_{3} \mathrm{SO}^{-}+\mathrm{HO}^{\bullet}$ & -588.31083 & -588.30537 & -588.41069 \\
\hline $\mathrm{CH}_{3} \mathrm{~S}^{-}+\mathrm{HO}_{2}^{\bullet}$ & -588.28265 & -588.27729 & -588.37837 \\
\hline $\mathrm{CH}_{3} \mathrm{~S}^{\bullet}+\mathrm{HO}_{2}^{-}$ & -588.25253 & -588.24992 & -588.36465 \\
\hline
\end{tabular}


Table 2. Gas phase potential energy $\left(\mathrm{E}_{\mathrm{p}, \mathrm{g}}\right)$, enthalpie $\left(\mathrm{H}_{\mathrm{g}}\right)$ and Gibbs free energy $\left(\mathrm{G}_{\mathrm{g}}\right)$ and aqueous solution Gibbs free energy $\left(\mathrm{G}_{\mathrm{aq}}\right)$ of the molecular states involved in methanethiol oxidation by the superoxide radical anion. All energies are given in Hartree.

\begin{tabular}{|c|c|c|c|c|}
\hline & $\mathrm{E}_{\mathrm{p}, \mathrm{g}}$ & $\mathrm{H}_{\mathrm{g}}$ & $\mathrm{G}_{\mathrm{g}}$ & $\mathrm{G}_{\mathrm{aq}}$ \\
\hline $\mathrm{RC}$ & -588.32886 & -588.26926 & -588.31056 & -588.39192 \\
\hline $\mathrm{TS} 1$ & -588.29630 & -588.23674 & -588.27445 & -588.36233 \\
\hline $\mathrm{AD}$ & -588.30055 & -588.23763 & -588.27557 & -588.36550 \\
\hline $\mathrm{TS} 2$ & -588.29449 & -588.23566 & -588.27317 & -588.36451 \\
\hline $\mathrm{PC}$ & -588.37627 & -588.31607 & -588.35316 & -588.44736 \\
\hline
\end{tabular}

Table 3. Gas phase potential energy $\left(\mathrm{E}_{\mathrm{p}, \mathrm{g}}\right)$, enthalpie $\left(\mathrm{H}_{\mathrm{g}}\right)$ and Gibbs free energy $\left(\mathrm{G}_{\mathrm{g}}\right)$ and aqueous solution Gibbs free energy $\left(\mathrm{G}_{\mathrm{aq}}\right)$ of the molecular states involved in methanethiol oxidation by the perhydroxyl radical. All energies are given in Hartree.

\begin{tabular}{|l|l|l|l|l|}
\hline & \multicolumn{1}{|c|}{$\mathrm{E}_{\mathrm{p}, \mathrm{g}}$} & \multicolumn{1}{c|}{$\mathrm{H}_{\mathrm{g}}$} & $\mathrm{G}_{\mathrm{g}}$ & $\mathrm{G}_{\mathrm{aq}}$ \\
\hline $\mathrm{CH}_{3} \mathrm{SH}+\mathrm{HO}_{2}{ }^{\circ}$ & -588.86618 & -588.79644 & -588.85109 & -588.85282 \\
\hline $\mathrm{RC}^{\prime}$ & -588.86977 & -588.79765 & -588.84144 & -588.83814 \\
\hline $\mathrm{TS} 1^{\prime}$ & -588.84701 & -588.77956 & -588.81784 & -588.81854 \\
\hline $\mathrm{PC}^{\prime}$ & -588.87939 & -588.80507 & -588.84729 & -588.84819 \\
\hline $\mathrm{TS}^{\prime}{ }^{\prime}$ & -588.82672 & -588.75639 & -588.79341 & -588.80125 \\
\hline $\mathrm{PC}^{\prime}$ & -588.98186 & -588.90776 & -588.95234 & -588.95761 \\
\hline $\mathrm{CH}_{3} \mathrm{~S}^{-}+\mathrm{H}_{2} \mathrm{O}_{2}$ & -588.86934 & -588.79923 & -588.85314 & -588.85714 \\
\hline $\mathrm{CH}_{3} \mathrm{SO}^{\bullet}+\mathrm{H}_{2} \mathrm{O}$ & -588.96778 & -588.89625 & -588.94959 & -588.95909 \\
\hline
\end{tabular}


